\title{
Sever Graves' disease-associated orbitopathy: a rare case of a frontal bone hemangioma.
}

\author{
Ahmad Alamri ${ }^{1}$, Daphné Pauchet ${ }^{1}$, André Balaton ${ }^{1}$, Constance Oriez ${ }^{1}$, Olivier Dupuy ${ }^{1}$, \\ and Adrien Ben Hamou ${ }^{2}$ \\ ${ }^{1}$ Paris Saint Joseph Hospital Group \\ ${ }^{2}$ Affiliation not available
}

June 30, 2020

\begin{abstract}
Bone hemangiomas are extremely rare benign tumors. We hereby present a case of 41-year old woman, that had a sever thyrotoxicosis, associated with severe GO which has necessitated glucocorticoid treatment. A link between hemangioma and sever thyrotoxicosis and/or IV GC might have resulted in endothelial alterations, and/or bone loss.
\end{abstract}

\section{Introduction}

Graves' disease (GD) is the most common cause of hyperthyroidism, affecting $1.5 \%$ of the population. It is more prevalent in women than in men $(\mathrm{F} / \mathrm{M} 7: 1)^{1}$. The most frequent extra-thyroidal manifestation is Graves' disease-associated orbitopathy (GO) affecting more females than males with an annual incidence of $0.016 \%$ and $0.003 \%$ respectively. This manifestation might lead to ocular adverse events as a corneal breakdown or an optic neuropathy in 3 to $5 \%$ of such patients. The European guidelines of Graves' diseaseassociated orbitopathy was commissioned by The European Thyroid Association to provide guidelines for the management of GO. They recommend a high-dose intravenous glucocorticoids (IV GC) be considered as a first-line treatment for moderate-to-severe and active $\mathrm{GO}^{2}$.

Skeletal hemangiomas are rare benign tumors of blood vessels which occur most commonly in the vertebral bodies followed by the skull. These tumors account for $0.7 \%$ of bone neoplasma ${ }^{3}$.

We hereby report a case of a severe GO treated by IV GC who developed a frontal bone hemangioma four months after the treatment.

\section{Case Report}

A 41 year-old Ivoirian woman, non-smoker, who has a history of a non-complicated type one diabetes, presented with GD, diagnosed in May 2018, with a clinical picture of weight loss and trembling. The initial biological tests showed an undetectable thyroid stimulating hormone (TSH) level, with high free thyroxine level (fT4) (31 pmol/L, N: 9.0-19.0 pmol/L), high free triiodothyronine (fT3) (11.8 pmol/L, N: 2.9-4.9 $\mathrm{pmol} / \mathrm{L}$ ), anti-thyrotropin receptor antibodies (TRAb) were positive at $54 \mathrm{IU} / \mathrm{l}(\mathrm{N}:<1.74 \mathrm{IU} / \mathrm{L})$, antithyroglobulin antibodies, and anti-thyroperoxidase antibodies were both negative. Alkaline phosphatase and bone turn-over markers were in the normal range excluding Paget's disease. Ultrasound showed a homogeneous hyper vascularized goiter with a peak systolic velocity estimated at $78 \mathrm{~cm} / \mathrm{s}(\mathrm{N}: 15-30 \mathrm{~cm} / \mathrm{s})$ with no other significant anomalies. A treatment with carbimazole was introduced. In December, during follow-up, the patient presented with GO that has developed over few months with conjunctival redness, chemosis, tearing, bilateral eyelid swelling, right upper eyelid retraction, blurry vision without diplopia, and ocular and retro orbital pain. Clinical activity score (CAS) was $7 / 10$ for both eyes. There was no optic nerve 
involvement. Due to the severity of her GO, she received a 10-week-course of high-dose IV GC pulses (six pulses of $750 \mathrm{mg}$ in 30 days, followed by 7 pulses of $430 \mathrm{mg}$ in about 45 days) to improve the GO, and to normalize her thyroid function test that remained uncontrolled despite high-doses of carbimazole. After the IV GC, clinical reassessment showed a mild to moderate improvement of the CAS of 4/10 in both eyes. TSH level remained undetectable, however fT4 and fT3 were normalized (10.4 and $4.6 \mathrm{pmol} / \mathrm{l}$ respectively). One month later, a total thyroidectomy was performed by the ENT team in May 2019, with no postoperative outcomes.

During the follow-up, the patient has signaled (August 2019) a frontal bone depression without any other symptoms or history of recent trauma. The frontal depression was left-sided, with no pain, tenderness, nor frontal paresthesia. The CT scan performed in November 2019 has showed a rounded lytic lesion of the frontal bone involving the walls of the frontal sinus, measuring $25 \times 25 \mathrm{~mm}$. The patient was then addressed to the ENT clinic, with a CT scan control after 3 months that showed an increase of the size of the lesion measuring $35 \times 42 \mathrm{~mm}$. Magnetic resonance imaging showed a single lytic lesion of the frontal bone left-sided with no intracranial invasion (Figure 1 ). This lesion was weakly hyper metabolic on the positron emission tomography (TEP) scan with a standardized uptake value $\left(\mathrm{SUV}_{\max }\right)$ of 4.8 .

A frontal bone biopsy was performed under local anesthesia. Histological examination showed remodeled bone trabeculae separated by a loose fibrous tissue containing numerous capillary vessels (Figure 2 ). Cytologic atypia, cell crowding, mitotic activity, and giant cells were not present. A diagnosis of intra-osseous hemangioma was established.

\section{Discussion}

Frontal bone hemangiomas are very rare tumors for which the origin is not yet clear. Some studies suggested a congenital origin - which remains doubtful since the mean age in most case reports was about 30 years while other studies reported a post-traumatic development ${ }^{4}$. However, no link between sever thyrotoxicosis and bone hemangiomas have been reported yet.

Firstly, changes in the systemic circulation - as may occur in venous obstruction and in heart failure - may result in changes in arterial and/or venous pressures which might explain the development of a vascular malformation. These changes might be the result of her sever thyrotoxicosis which has persisted for more than a year.

Secondly, the possible link between the lesion and high-dose IV GC administration in a short period (7.5 grams in 10 weeks). To our knowledge, no cases of hemangiomas resulting from high-dose of GC therapy have been described ${ }^{5}$. We propose two mechanisms: $1 /$ circulatory changes resulting from high-dose IV GC, which could be explained by the expression of both type 1 and type $211 \beta$-hydroxysteroid dehydrogenase isozymes in the arterial wall; which might have a direct impact on vascular physiology, 2/ the increased risk of bone loss associated with the IV GC therapy; as a result of (i) a decrease in bone formation, by directly inhibiting osteoblastic proliferation and differentiation, and by increasing the apoptosis of mature osteoblasts, (ii) an increase in bone resorption by stimulating osteoclastogenesis. According to the literature, this bone loss caused by high-dose GC therapy is most pronounced in the first months following a high-dose GC therapy, and the fact that the lesion appeared four months after the IV GC might suggest a physiopathological link.

In conclusion, frontal bone hemangiomas are rare lesions, for which the etiology is not clear. To our knowledge, there are no studies which have described the occurrence of bone hemangiomas in patients having severe GO, or in patients treated with high-dose IV GC. We suggest a possible link between IV GC which might have resulted in endothelial alterations, and/or bone loss. Severe thyrotoxicosis might have contributed due to possible circulatory alterations. Finally, the role of carbimazole cannot be excluded despite the lack of evidence.

\section{References}

1. Smith TJ, Hegedüs L. Graves' Disease. N Engl J Med . 2016;375(16):1552-1565. 
2. Bartalena L, Baldeschi L, Boboridis K, et al. The 2016 European Thyroid Association/European Group on Graves' Orbitopathy Guidelines for the Management of Graves' Orbitopathy. Eur Thyroid J . 2016;5(1):9-26.

3. Seo BF, Kang KJ, Jung S-N, Byeon JH. Skeletal cavernous hemangiomas of the frontal bone with orbital roof and rim involvement. Arch Craniofac Surg . 2018;19(3):214-217.

4. Cheng N-C, Lai D-M, Hsie M-H, Liao S-L, Chen Y-BT. Intraosseous hemangiomas of the facial bone. Plast Reconstr Surg . 2006;117(7):2366-2372.

5. Ueda-Sakane Y, Kanamoto N, Fushimi Y, et al. Overall safety and efficacy of high-dose and low-dose intravenous glucocorticoid therapy in patients with moderate-to-severe active Graves' ophthalmopathy.Endocr $J$. 2016;63(8):703-714.

\section{Legends}

Figure 1. The lytic bone lesion on computed tomography scan (CT) and magnetic resonance imaging (MRI).

1. CT scan showing a rounded lytic lesion of the frontal bone, left-sided, involving the walls of the frontal sinus, measuring $35 \times 42 \mathrm{~mm}$ in diameter.

2. Magnetic resonance imaging showing the frontal bone lesion, lateralized to the left, with hyposignal in T1-weighted sequences, enhanced after injection with gadolinium.

Figure 2. Numerous capillary vessels containing erythrocytes, in between pre-existing bone trabeculae (Haematoxylin-eosin; original magnification, X 250).

\section{Hosted file}

FIGURE_1.docx available at https://authorea.com/users/338463/articles/464464-sever-gravesdisease-associated-orbitopathy-a-rare-case-of-a-frontal-bone-hemangioma

\section{Hosted file}

FIGURE_2.docx available at https://authorea.com/users/338463/articles/464464-sever-gravesdisease-associated-orbitopathy-a-rare-case-of-a-frontal-bone-hemangioma 